\title{
Synthesis of Pyronyl Derivatives as Resveratrol Analogues and Their Inhibitory Effects on Nitric Oxide and $\mathrm{PGE}_{2}$ Productions
}

\author{
Min Hwan Kim, Ji-Sun Shin, Kyung-Tae Lee, and Yong Sup Lee* \\ Department of Pharmaceutical Science \& Department of Life and Nanopharmaceutical Science, College of Pharmacy, \\ Kyung Hee University, 1 Hoegi-dong, Dongdaemoon-ku, Seoul 130-701, Korea. ${ }^{*}$ E-mail: kyslee@khu.ac.kr \\ Received July 6, 2010, Accepted September 15, 2010
}

Key Words: Resveratrol, Pyrone, Nitrc oxide, Prostaglandin E2, Anti-inflammatory

Inflammation is one of the most important aspects of host defense mechanisms against invading pathogens. However, inflammation may also aid microbial pathogenesis because the inflammatory response elicited by an invading microorganism can result in considerable host damage, making nutrients available and providing access to host tissues.

Nitric oxide (NO) and prostaglandins (PGs) are two major mediators produced at inflammatory sites by the enzymes such as $i$ NOS (inducible nitric oxide synthase) and COX-2. In inflammation, overproduction of NO is mainly caused by $i$ NOS, which is up-regulated in macrophages by cytokine and/or bacterial lipopolysaccharide (LPS) stimulation. ${ }^{2}$ Despite its beneficial role in host defense, sustained NO production can be deleterious to the host and has been implicated in the pathogenesis of various inflammatory diseases. ${ }^{3} \mathrm{COX}-2$ is also one of enzymes that participate in inflammation, and induction of COX-2 is responsible for the production of $\mathrm{PGE}_{2}$ at the site of inflammation. ${ }^{4}$ Therefore, the regulations of $\mathrm{NO}$ and $\mathrm{PGE}_{2}$ productions have been current research topics for the new anti-inflammatory drugs development.

Resveratrol (1), a polyphenolic phytochemical found in grapes, ${ }^{5}$ blueberries, ${ }^{6}$ and peanuts, ${ }^{7}$ has been found to have a wide range of pharmacological activities, such as antioxidant, anti-inflammatory, antitumour and immunomodulatory activities. Previous studies have shown that resveratrol exhibit antiinflammatory activities through the non-selective inhibition of COX-2 and COX-1..$^{8-9}$ Resveratrol has also been found to suppress macrophage activation and controls the expression of $i$ NOS which accounts for its anti-inflammatory effects. ${ }^{10-11}$ However, due to its low oral bioavailability ${ }^{12}$ and the relatively high concentrations of resveratrol which are required to exert anti-inflammatory activity, ${ }^{13}$ the modifications of the structure of resveratrol were desired to produce more efficient anti-inflammatory agents. In fact, there has been a number of reports on the synthesis of resveratrol analogues, such as hydroxystilbenes, ${ }^{14-17}$ styrylthiophenes, styrylpyridines, and styrylquinolines. ${ }^{18}$ We also reported the synthesis of styrylquinazolines as resveratrol analogues and their ability to inhibit the production of $\mathrm{PGE}_{2}$ in LPS-activated macrophage cells. ${ }^{19}$

In the present study, pyronyl-vinyl derivatives $\mathbf{2}$ were prepared as resveratrol analogues and evaluated for their ability to inhibit both enzymes using in vitro assay for $i$ NOS and COX-2 by measuring $\mathrm{NO}$ and $\mathrm{PGE}_{2}$ productions, respectively, by LPSactivated macrophage cells. We envisioned that the substituted

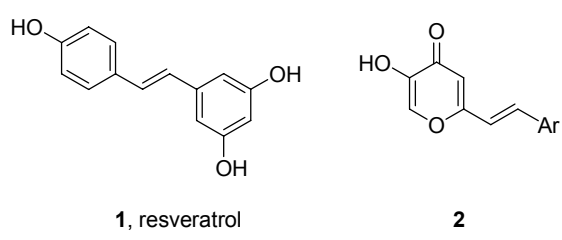

pyran ring in $\mathbf{2}$ can be considered as hydroxylated benzene in resveratrol as was proven in our synthesis of tyrosinase inhibitory (4-oxo-4H-pyran-2-yl)acrylic acid as a caffeic or ferulic acid mimic. $^{20}$

Chemistry. The synthesis of pyronyl-vinyl derivatives was accomplished as shown in Scheme 1. For the construction of the trans-olefins, Horner-Emmons-Wadsworth reaction was utilized. ${ }^{21}$ Reaction of pyronyl phosphonate $\mathbf{3}^{19}$ with aromatic carboxaldehydes in THF using $\mathrm{NaH}$ as the base afforded PMBprotected pyronyl-vinyl derivatives $\mathbf{4 a - 4 j}$. Several systems such as $\mathrm{AcCl} /$ ethanol, ${ }^{22}$ trifluoroacetic acid $/ \mathrm{CH}_{2} \mathrm{Cl}_{2},{ }^{23}$ cerium (IV) ammonium nitrate, ${ }^{24}$ and $\mathrm{DDQ}^{25}$ were tried for the deprotection of PMB group in $\mathbf{4 a - 4 j}$ without success. Finally, it was found that the use of $\mathrm{BBr}_{3}$ was effective for clean removal of $\mathrm{PMB}$ group. The $\mathrm{PMB}$ protecting group on compounds $\mathbf{4 a - 4 j}$ was removed using $\mathrm{BBr}_{3}$ in $\mathrm{CH}_{2} \mathrm{Cl}_{2}$ to give eight pyronylvinyl derivatives $\mathbf{2 a - 2} \mathbf{j}$ in moderate to good yields. ${ }^{26}$ For the synthesis of hydroxylated benzene-substituted pyronyl-vinyl derivatives $\mathbf{2 b}$ and 2c, acetoxybenzene carboxaldehydes were used in the Horner-Emmons-Wadsworth reaction. Acetyl protecting groups were simultaneously removed during the deprotection step of PMB group.

Biological evaluation. The synthesized pyronyl-vinyl derivatives $\mathbf{2 a - 2} \mathbf{h}$ were evaluated for their ability to inhibit the LPSactivated production of the inflammatory mediators, $\mathrm{NO}$ and $\mathrm{PGE}_{2}$ in RAW 264.7 cells. The screen for activity was performed
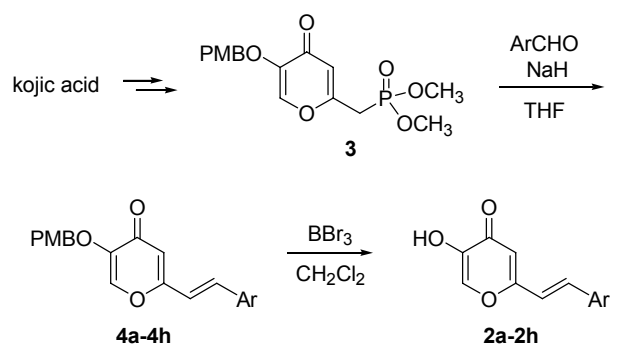

Scheme 1 
Table 1. Chemical yields, NO and $\mathrm{PGE}_{2}$ production inhibition, and cytotoxicity of pyronyl-vinyl derivatives 4a-4h on LPS-activated RAW 264.7 macrophage cells

\begin{tabular}{|c|c|c|c|c|c|}
\hline \multirow{3}{*}{ Compds } & & & \multirow{2}{*}{\multicolumn{3}{|c|}{$\mathrm{IC}_{50}(\mu \mathrm{M})^{a}$}} \\
\hline & \multirow{2}{*}{$\mathrm{Ar}$} & \multirow{2}{*}{$\begin{array}{l}\text { Overall yields }(\%) \\
\quad \text { from } 3\end{array}$} & & & \\
\hline & & & NO & $\mathrm{PGE}_{2}$ & Cytotoxicity \\
\hline $2 \mathbf{a}$ & phenyl & 52 & $84.29 \pm 6.44$ & $47.40 \pm 2.76$ & $157.69 \pm 5.10$ \\
\hline $2 \mathbf{b}$ & 4-hydroxyphenyl & 33 & $45.01 \pm 1.59$ & $3.20 \pm 0.00$ & $81.62 \pm 11.54$ \\
\hline $2 c$ & 3,4-dihydroxyphenyl & 37 & $31.11 \pm 2.51$ & $10.27 \pm 0.32$ & $71.15 \pm 6.26$ \\
\hline 2d & 4-bromophenyl & 45 & $23.23 \pm 8.71$ & $11.49 \pm 2.82$ & $25.97 \pm 3.41$ \\
\hline $2 e$ & 4-nitrophenyl & 77 & $33.19 \pm 4.61$ & $87.99 \pm 8.46$ & $73.62 \pm 14.10$ \\
\hline $2 f$ & 2-pyridyl & 48 & $52.05 \pm 4.06$ & $76.40 \pm 2.02$ & $153.38 \pm 1.78$ \\
\hline $2 \mathrm{~g}$ & 2-furyl & 13 & $37.94 \pm 3.15$ & $32.92 \pm 1.33$ & $95.02 \pm 20.41$ \\
\hline $2 \mathrm{~h}$ & 2-thienyl & 43 & $41.03 \pm 1.79$ & $49.10 \pm 0.39$ & $150.92 \pm 17.51$ \\
\hline 1, resveratrol & & & $45.65 \pm 2.87$ & $5.69 \pm 1.82$ & $21.81 \pm 4.46$ \\
\hline L-NIL & & & $9.50 \pm 5.89$ & - & - \\
\hline NS-398 & & & - & $2.20 \pm 1.25$ & - \\
\hline
\end{tabular}

${ }^{a} \mathrm{IC}_{50}$ is defined as the concentration that results in a $50 \%$ inhibition. Data are presented as the means \pm S.D. of three independent experiments.

in a dose-response to determine the $\mathrm{IC}_{50}$ value. The results are summarized in Table 1. Resveratrol was used as a reference for comparisons. L- $N^{6}$-(1-iminoethyl) lysine (L-NIL, $10 \mu \mathrm{M}$ ) and NS-398 $(5 \mu \mathrm{M})$ were also used as an inhibitor of NO accumulation and a COX-2 selective inhibitor for $\mathrm{PGE}_{2}$ production, respectively. The cytotoxic effects of compounds were also evaluated in the presence or absence of LPS using the MTT assay to test whether the $\mathrm{NO}$ and $\mathrm{PGE}_{2}$ production inhibitory effects observed were attributable to cytotoxic effects. Generally, pyronyl-vinyl derivatives exhibited similar level of NO production and enhanced $\mathrm{PGE}_{2}$ production inhibitory activities on RAW264.7 cells when compared to those of the parent compound, resveratrol (1). On the other hands, every compound except 2c showed $3-7$ times less cytotoxicities on tested cells than 1 indicating that the change of phenyl ring in resveratrol into pyrone ring influenced on the cytotoxicity. Compounds $\mathbf{2 b}$ and $\mathbf{2 c}$, which have a hydroxyl-substituted benzene ring showed the most potent $\mathrm{PGE}_{2}$ production inhibitory activities with $\mathrm{IC}_{50}$ values of 3.2 and $10.27 \mu \mathrm{M}$ with similar level of NO production inhibitory activities to that of resveratrol. Introduction of heterocycles (2f-2h) also retained NO production inhibition activity, but reduced $\mathrm{PGE}_{2}$ production inhibition effects.

In conclusion, pyronyl-vinyl derivatives $\mathbf{2}$ were prepared as resveratrol analogues through the Horner-Emmons-Wadsworth reaction of pyronyl phosphonate $\mathbf{3}$ with various aromatic carboxaldehydes and evaluated for their ability to inhibit $\mathrm{NO}$ and $\mathrm{PGE}_{2}$ productions on LPS-activated macrophage cells. Among synthesized, compound $\mathbf{2 b}$ showed improved $\mathrm{NO}$ and $\mathrm{PGE}_{2}$ production inhibitory activities on LPS-activated RAW264.7 cells with less cytotoxicity than parent compound, resveratrol.

\section{Experimental Section}

Materials and chemicals. Dulbecco's modified Eagle's minimum essential medium (DMEM), fetal bovine serum (FBS), penicillin, and streptomycin were obtained from Life Technologies Inc. (Grand Island, NY, USA). The enzyme immunoassay (EIA) kit for $\mathrm{PGE}_{2}$ was obtained from R\&D Systems
(Minneapolis, MN, USA). 3-(4,5-dimethylthiazol-2-yl)-2,5diphenyl tetrazolium bromide (MTT), L- $N^{6}$-(1-iminoethyl)lysine (L-NIL), NS-398, lipopolysaccharide (LPS) (Escherichia coli, serotype 0111:B4) and all other chemicals were purchased from the Sigma Chemical Co. (St. Louis, MO, USA). ${ }^{1} \mathrm{H}$ and ${ }^{13} \mathrm{C}$ NMR spectra were recorded on a Gemini Varian-400 (400 and $100 \mathrm{MHz}$, respectively). Analytical thin layer chromatographies (TLC) were carried out by pre-coated silica gel (E. Merck, Kiesegel 60F254 layer thickness $0.25 \mathrm{~mm}$ ). All solvents used were purified according to standard procedures.

General procedure for the synthesis of $4 \mathrm{a}-4 \mathrm{~h}$. To a stirred suspension of $\mathrm{NaH}$ (1.5 eq.) in THF was added dropwise a solution of dimethyl [5-(4-methoxybenzyloxy)-4-oxo-4H-pyran-2-yl]methylphosphonate ${ }^{19}(3,1$ eq. $)$ in THF at $0{ }^{\circ} \mathrm{C}$ and stirred for $20 \mathrm{~min}$. The mixture was treated with aromatic carboxaldehyde (1.2 eq.) at the same temperature, warmed up to room temperature, and further stirred for $2 \mathrm{~h}$. The reaction mixture was diluted with EtOAc and washed with water. The organic layer was dried over $\mathrm{MgSO}_{4}$, concentrated, and purified by crystallization or flash column chromatography to afford 4a-4h.

(E)-5-(4-Methoxybenzyloxy)-2-styryl-4H-pyran-4-one (4a): The compound $4 \mathrm{a}(251 \mathrm{mg})$ was obtained according to the above general procedure from the compound $3(300 \mathrm{mg}, 0.84 \mathrm{mmol})$ and benzaldehyde $(0.1 \mathrm{~mL}, 0.93 \mathrm{mmol})$. Yield: $89 \%$; ${ }^{1} \mathrm{H}$ NMR $\left(\mathrm{CD}_{3} \mathrm{OD}\right) \delta 8.01(\mathrm{~s}, 1 \mathrm{H}$, pyrone- $H 6), 7.64-7.59(\mathrm{~m}, 2 \mathrm{H}$, aromatic), 7.47 (d, $1 \mathrm{H}, J=16.2 \mathrm{~Hz},-\mathrm{CH}=\mathrm{CH}-), 7.40-7.35$ (m, 4H, aromatic), $6.96(\mathrm{~d}, 1 \mathrm{H}, J=16.2 \mathrm{~Hz},-\mathrm{CH}=\mathrm{CH}-), 6.93(\mathrm{~d}, 2 \mathrm{H}$, $J=8.6 \mathrm{~Hz}$, aromatic), 6.51 (s, $1 \mathrm{H}$, pyrone- $H 3), 4.98(\mathrm{~s}, 2 \mathrm{H}$, $\left.-\mathrm{OCH}_{2} \mathrm{Ar}\right), 3.79$ (s, $\left.3 \mathrm{H},-\mathrm{OCH}_{3}\right)$.

(E)-2-[2-(4-Acetoxyphenyl)vinyl]-5-(4-methoxybenzyloxy)4H-pyran-4-one (4b): The compound $4 \mathrm{~b}(225 \mathrm{mg})$ was obtained according to the above general procedure from the compound $3(300 \mathrm{mg}, 0.84 \mathrm{mmol})$ and 4-acetoxybenzaldehyde (153 mg, 0.93 mmol). Yield: 67\%; ${ }^{1} \mathrm{H}$ NMR $\left(\mathrm{CDCl}_{3}\right) \delta 7.53$ (s, $1 \mathrm{H}$, pyrone- $H 6), 7.50(\mathrm{~d}, 2 \mathrm{H}, J=8.4 \mathrm{~Hz}$, aromatic), 7.35-7.31 $(\mathrm{m}, 3 \mathrm{H}$, aromatic, $-\mathrm{CH}=\mathrm{CH}-), 7.12(\mathrm{~d}, 2 \mathrm{H}, J=10.2 \mathrm{~Hz}$, aromatic), $6.89(\mathrm{~d}, 2 \mathrm{H}, J=8.4 \mathrm{~Hz}$, aromatic), $6.60(\mathrm{~d}, 1 \mathrm{H}, J=16.2 \mathrm{~Hz}$, 
$-\mathrm{CH}=\mathrm{CH}-), 6.41$ (s, $1 \mathrm{H}$, pyrone- $H 3), 5.04$ (s, 2H, $\left.-\mathrm{OCH}_{2} \mathrm{Ar}\right)$, $3.80\left(\mathrm{~s}, 3 \mathrm{H},-\mathrm{OCH}_{3}\right), 2.03\left(\mathrm{~s}, 3 \mathrm{H}, \mathrm{CH}_{3} \mathrm{CO}_{2-}\right)$.

(E)-2-[2-(3,4-Diacetoxyphenyl)vinyl]-5-(4-methoxybenzyloxy)-4H-pyran-4-one (4c): The compound 4c (1.61 g) was obtained according to the above general procedure from the compound 3 ( $2 \mathrm{~g}, 5.64 \mathrm{mmol}$ ) and 3,4-diacetoxybenzaldehyde (1.38 g, $6.2 \mathrm{mmol})$. Yield: $63 \% ;{ }^{1} \mathrm{H} \mathrm{NMR}\left(\mathrm{CDCl}_{3}\right) \delta 7.52(\mathrm{~s}, 1 \mathrm{H}$, pyrone-H6), 7.35-7.21 (m, 6H, aromatic, $-\mathrm{CH}=\mathrm{CH}-), 6.90$ (d, $2 \mathrm{H}, J=8.6 \mathrm{~Hz}, \mathrm{Ar}-H 6), 6.87$ (d, 2H, aromatic), 6.58 (d, 1H, $J=$ $15.9 \mathrm{~Hz},-\mathrm{CH}=\mathrm{CH}-), 6.37$ (s, 1H, pyrone- $H 3), 5.04(\mathrm{~s}, 2 \mathrm{H}$, $\left.-\mathrm{OCH}_{2} \mathrm{Ar}\right), 3.80\left(\mathrm{~s}, 3 \mathrm{H},-\mathrm{OCH}_{3}\right), 2.31\left(\mathrm{~s}, 3 \mathrm{H}, \mathrm{CH}_{3} \mathrm{CO}_{2}-\right), 2.30$ (s, $3 \mathrm{H}, \mathrm{CH}_{3} \mathrm{CO}_{2}-$ ).

(E)-2-(4-Bromostyryl)-5-(4-methoxybenzyloxy)-4H-pyran4-one (4d): The compound 4d (257 mg) was obtained according to the above general procedure from the compound 3 (300 $\mathrm{mg}, 0.84 \mathrm{mmol}$ ) and 4-bromobenzaldehyde (172 $\mathrm{mg}, 0.93$ mmol). Yield: $72 \% ;{ }^{1} \mathrm{H}$ NMR $\left(\mathrm{CDCl}_{3}\right) \delta 7.53-7.21(\mathrm{~m}, 7 \mathrm{H}$, aromatic, $-\mathrm{CH}=\mathrm{CH}-$, pyrone- $H 6), 6.94(\mathrm{~d}, 1 \mathrm{H}, J=8.4 \mathrm{~Hz}$, aromatic), $6.64(\mathrm{~d}, 1 \mathrm{H}, J=16.2 \mathrm{~Hz},-\mathrm{CH}=\mathrm{CH}-), 6.64(\mathrm{~s}, 1 \mathrm{H}$, pyrone- $H 3)$, 5.04 (s, $\left.2 \mathrm{H},-\mathrm{OCH} \mathrm{H}_{2} \mathrm{Ar}\right), 3.80\left(\mathrm{~s}, 3 \mathrm{H},-\mathrm{OCH}_{3}\right)$.

(E)-5-(4-Methoxybenzyloxy)-2-(4-nitrostyryl)-4H-pyran4-one (4e): The compound $4 \mathrm{e}(282 \mathrm{mg}$ ) was obtained according to the above general procedure from the compound $\mathbf{3}(300 \mathrm{mg}$, $0.84 \mathrm{mmol})$ and 4-nitrobenzaldehyde $(140 \mathrm{mg}, 0.93 \mathrm{mmol})$. Yield: $87 \%$; ${ }^{1} \mathrm{H}$ NMR $\left(\mathrm{CDCl}_{3}\right) \delta 8.28(\mathrm{~d}, 2 \mathrm{H}, J=8.7 \mathrm{~Hz}$, aromatic), 8.26 (s, $1 \mathrm{H}$, pyrone-H6), 8.21 (d, $2 \mathrm{H}, J=8.7 \mathrm{~Hz}$, aromatic), 7.55 (d, $1 \mathrm{H}, J=16.5 \mathrm{~Hz},-\mathrm{CH}=\mathrm{CH}-), 7.39-7.30(\mathrm{~m}, 3 \mathrm{H}$, aromatic, $-\mathrm{CH}=\mathrm{CH}-), 6.98(\mathrm{~d}, 2 \mathrm{H}, J=8.7 \mathrm{~Hz}$, aromatic), $6.60(\mathrm{~s}$, $1 \mathrm{H}$, pyrone- $\mathrm{H3}), 4.91$ (s, $\left.2 \mathrm{H},-\mathrm{OCH} \mathrm{H}_{2} \mathrm{Ar}\right), 3.77$ (s, 3H, $\left.-\mathrm{OCH}_{3}\right)$.

(E)-5-(4-Methoxybenzyloxy)-2-[2-(pyridin-2-yl)vinyl]-4Hpyran-4-one (4f): The compound $\mathbf{4 f}(167 \mathrm{mg})$ was obtained according to the above general procedure from the compound 3 (300 mg, $0.84 \mathrm{mmol}$ ) and picolinaldehyde $(0.08 \mathrm{~mL}, 0.93$ mmol). Yield: $58 \%$; ${ }^{1} \mathrm{H}$ NMR $\left(\mathrm{CDCl}_{3}\right) \delta 8.64(\mathrm{~d}, 1 \mathrm{H}, J=6.0 \mathrm{~Hz}$, pyridin-H6), $7.72(\mathrm{~m}, 1 \mathrm{H}$, pyridin- $H 4), 7.55(\mathrm{~s}, 1 \mathrm{H}$, pyrone- $H 6)$, 7.73-7.23 (m, $6 \mathrm{H}$, aromatic, $-\mathrm{CH}=\mathrm{CH}-), 6.89(\mathrm{~d}, 2 \mathrm{H}, J=8.8 \mathrm{~Hz}$, aromatic), $6.46(\mathrm{~s}, 1 \mathrm{H}$, pyrone- $\mathrm{H3}), 5.04\left(\mathrm{~s}, 2 \mathrm{H},-\mathrm{OCH} \mathrm{H}_{2} \mathrm{Ar}\right)$, $3.80\left(\mathrm{~s}, 3 \mathrm{H}, \mathrm{CH}_{3}\right)$.

(E)-2-(2-(Furan-2-yl)vinyl)-5-(4-methoxybenzyloxy)-4Hpyran-4-one (4g): The compound $\mathbf{4 g}(111 \mathrm{mg})$ was obtained according to the above general procedure from the compound 3 (300 mg, $0.84 \mathrm{mmol}$ ) and furan-2-carbaldehyde ( $89 \mathrm{mg}, 0.93$ mmol). Yield: $40 \% ;{ }^{1} \mathrm{H}$ NMR $\left(300 \mathrm{MHz}, \mathrm{CDCl}_{3}\right) \delta 8.13(\mathrm{~s}, 1 \mathrm{H}$, pyrone-Ho), 7.83 (br s, $1 \mathrm{H}$, furan-H5) $7.36(\mathrm{~d}, 2 \mathrm{H}, J=8.7 \mathrm{~Hz}$, aromatic) $7.24(\mathrm{~d}, 1 \mathrm{H}, J=16.2 \mathrm{~Hz},-\mathrm{CH}=\mathrm{CH}-), 6.96(\mathrm{~d}, 2 \mathrm{H}, J=$ $8.7 \mathrm{~Hz}$, aromatic) $6.83(\mathrm{~d}, 1 \mathrm{H}, J=3.2 \mathrm{~Hz}$, furan- $H 3), 6.75(\mathrm{~d}$, $1 \mathrm{H}, J=16.2 \mathrm{~Hz},-\mathrm{CH}=\mathrm{CH}-), 6.63-6.65(\mathrm{~m}, 1 \mathrm{H}$, furan- $H 4), 6.53$ (s, $1 \mathrm{H}$, pyrone- $\mathrm{H3}), 4.89$ (s, $\left.2 \mathrm{H},-\mathrm{OCH} \mathrm{H}_{2} \mathrm{Ar}\right), 3.77$ (s, $\left.3 \mathrm{H},-\mathrm{OCH}_{3}\right)$.

(E)-5-(4-Methoxybenzyloxy)-2-(2-(thiophen-2-yl)vinyl)4H-pyran-4-one (4h): The compound $4 \mathrm{~h}(256 \mathrm{mg})$ was obtained according to the above general procedure from the compound 3 (300 $\mathrm{mg}, 0.84 \mathrm{mmol}$ ) and thiophene-2-carbaldehyde (104 mg, $0.93 \mathrm{mmol}$ ). Yield: 80\%; ${ }^{1} \mathrm{H}$ NMR (DMSO- $\left.d_{6}\right) \delta 8.12$ (s, $1 \mathrm{H}$, pyrone-H6), $7.66(\mathrm{~d}, 1 \mathrm{H}, J=4.6 \mathrm{~Hz}$, thiophene- $H 5)$, 7.57 (d, $1 \mathrm{H}, J=16.2 \mathrm{~Hz},-\mathrm{CH}=\mathrm{CH}-), 7.43$ (d, $1 \mathrm{H}, J=3.6 \mathrm{~Hz}$, thiophene-H3), $7.35(\mathrm{~d}, 2 \mathrm{H}, J=8.4 \mathrm{~Hz}, \mathrm{Ph}-H 2, H 6), 7.13(\mathrm{~m}$, $1 \mathrm{H}$, thiophene- $\mathrm{H4}), 6.92-6.97(\mathrm{~m}, 2 \mathrm{H}$, aromatic $), 6.76(\mathrm{~d}, 1 \mathrm{H}$, $J=16.2 \mathrm{~Hz},-\mathrm{CH}=\mathrm{CH}-), 6.49$ (s, $1 H$, pyrone- $H 3), 4.88(\mathrm{~s}, 2 \mathrm{H}$,
$\left.-\mathrm{OCH}_{2} \mathrm{Ar}\right), 3.76$ (s, 3H, $-\mathrm{OCH}_{3}$ ).

General procedure for the synthesis of (2a-2h). To a solution of $\mathbf{4 a - 4 h}$ (1.0 eq.) in $\mathrm{CH}_{2} \mathrm{Cl}_{2}(5 \mathrm{~mL})$ was added $\mathrm{BBr}_{3}(1 \mathrm{M}$ in $\mathrm{CH}_{2} \mathrm{Cl}_{2}, 10$ eq.), and the resultant mixture was stirred for 12 $\mathrm{h}$ at room temperature. The mixture was treated with ice-cold methanol $(10 \mathrm{~mL})$ and stirred at $15{ }^{\circ} \mathrm{C}$ for $30 \mathrm{~min}$. The solvent was removed in vacuo, and the addition of fresh methanol $(3 \times$ $5 \mathrm{~mL}$ ) followed by evaporation was repeated. The residue was treated with ethanol and the resulting precipitate was filtered, washed with ethanol, and dried to provide $\mathbf{2} \mathbf{a}-\mathbf{2} \mathbf{h}$.

(E)-5-Hydroxy-2-styryl-4H-pyran-4-one (2a): The compound $\mathbf{2 a}(73 \mathrm{mg})$ was obtained according to the above general procedure from the compound $3(200 \mathrm{mg}, 0.59 \mathrm{mmol})$. Yield: $57 \% ;{ }^{1} \mathrm{H}$ NMR (DMSO- $d_{6}$ ) $\delta 7.98$ (s, $1 \mathrm{H}$, pyrone-H6), 7.63-7.59 (m, 2H, aromatic), $7.47(\mathrm{~d}, 1 \mathrm{H}, \mathrm{J}=16.2 \mathrm{~Hz},-\mathrm{CH}=\mathrm{CH}-), 7.40-$ 7.35 (m, 3H, aromatic), 6.96 (d, $1 \mathrm{H}, J=16.2 \mathrm{~Hz},-\mathrm{CH}=\mathrm{CH}-$ ), 6.50 (s, $1 \mathrm{H}$, pyrone- $H 3)$.

(E)-5-Hydroxy-2-(4-hydroxystyryl)-4H-pyran-4-one (2b): The compound $\mathbf{2 b}(40 \mathrm{mg})$ was obtained according to the above general procedure from the compound $3(200 \mathrm{mg}, 0.50 \mathrm{mmol})$. Yield: $34 \%$; ${ }^{1} \mathrm{H}$ NMR (DMSO- $\left.d_{6}\right) \delta 8.01(\mathrm{~s}, 1 \mathrm{H}$, pyrone-Ho), 7.49 (d, $2 \mathrm{H}, J=8.6 \mathrm{~Hz}$, aromatic), 7.29 (d, $1 \mathrm{H}, J=16.4 \mathrm{~Hz}$, $-\mathrm{CH}=\mathrm{CH}-), 6.82(\mathrm{~d}, 1 \mathrm{H}, J=16.4 \mathrm{~Hz},-\mathrm{CH}=\mathrm{CH}-), 6.79(\mathrm{~d}, 2 \mathrm{H}$, $J=8.6 \mathrm{~Hz}$, aromatic), 6.41 (s, $1 \mathrm{H}$, pyrone- $H 3) ;{ }^{13} \mathrm{C} \mathrm{NMR}$ $\left(\mathrm{DMSO}-d_{6}\right) \delta 174.3,162.1,159.4,146.2,139.2,135.5,129.8$, $126.5,116.9,111.2$.

(E)-2-(3,4-Dihydroxystyryl)-5-hydroxy-4H-pyran-4-one (2c): The compound $2 \mathrm{c}(67 \mathrm{mg})$ was obtained according to the above general procedure from the compound 3 (200 mg, 0.44 mmol). Yield: $61 \%$; ${ }^{1} \mathrm{H}$ NMR (DMSO- $\left.d_{6}\right) \delta 8.04$ (s, $1 \mathrm{H}$, pyrone-H6), $7.21(\mathrm{~d}, 1 \mathrm{H}, J=16.2 \mathrm{~Hz},-\mathrm{CH}=\mathrm{CH}-), 7.02(\mathrm{~d}, 1 \mathrm{H}, J=$ $0.8 \mathrm{~Hz}, \mathrm{Ar}-H 2), 6.93$ (dd, $1 \mathrm{H}, J=8.4,0.8 \mathrm{~Hz}, \mathrm{Ar}-H 6), 6.77$ (d, $1 \mathrm{H}, J=8.4 \mathrm{~Hz}, \mathrm{Ar}-H 5), 6.20$ (d, $1 \mathrm{H}, J=16.2 \mathrm{~Hz},-\mathrm{CH}=\mathrm{CH}-)$, 6.44 (s, $1 \mathrm{H}$, pyrone- $H 3) ;{ }^{13} \mathrm{C}$ NMR (DMSO- $\left.d_{6}\right) \delta 174.3,162.1$, $147.9,146.1,146.0,139.1,135.8,127.0,120.9,116.7,116.2$, $114.5,111.2$.

(E)-2-(4-Bromostyryl)-5-hydroxy-4H-pyran-4-one (2d): The compound $2 \mathbf{d}(88 \mathrm{mg}$ ) was obtained according to the above general procedure from the compound $3(200 \mathrm{mg}, 0.48 \mathrm{mmol})$. Yield: $62 \% ;{ }^{1} \mathrm{H}$ NMR (DMSO- $\left.d_{6}\right) \delta 8.06(\mathrm{~s}, 1 \mathrm{H}$, pyrone- $H 6)$, 7.62 (br s, 4H, aromatic), 7.37 (d, $1 \mathrm{H}, J=16.4 \mathrm{~Hz},-\mathrm{CH}=\mathrm{CH}-$ ), $7.12(\mathrm{~d}, 1 \mathrm{H}, J=16.4 \mathrm{~Hz},-\mathrm{CH}=\mathrm{CH}-), 6.51(\mathrm{~s}, 1 \mathrm{H}$, pyrone- $H 3)$; ${ }^{13} \mathrm{C} \mathrm{NMR}\left(\mathrm{CDCl}_{3}\right) \delta 174.7,161.4,146.8,139.8,135.1,134.2$, 132.6, 130.2, 123.4, 121.7, 113.0.

(E)-5-Hydroxy-2-(4-nitrostyryl)-4H-pyran-4-one (2e): The compound $2 \mathbf{e}(120 \mathrm{mg})$ was obtained according to the above general procedure from the compound $3(200 \mathrm{mg}, 0.44 \mathrm{mmol})$. Yield: $88 \%$; ${ }^{1} \mathrm{H}$ NMR (DMSO- $\left.d_{6}\right) \delta 8.26(\mathrm{~d}, 2 \mathrm{H}, J=8.6 \mathrm{~Hz}$, aromatic), $8.10(\mathrm{~s}, 1 \mathrm{H}$, pyrone- $H 6), 7.92(\mathrm{~d}, 2 \mathrm{H}, J=8.6 \mathrm{~Hz}$, aromatic), $7.51(\mathrm{~d}, 1 \mathrm{H}, J=16.4 \mathrm{~Hz},-\mathrm{CH}=\mathrm{CH}-), 7.34(\mathrm{~d}, 1 \mathrm{H}, J=16.4$ $\mathrm{Hz},-\mathrm{CH}=\mathrm{CH}-), 6.59(\mathrm{~s}, 1 \mathrm{H}$, pyrone- $H 3) ;{ }^{13} \mathrm{C}$ NMR (DMSO- $d_{6}$ ) $\delta 174.6,160.8,148.1,147.0,142.4,140.1,133.0,129.2,125.2$, 124.8, 114.1 .

(E)-5-Hydroxy-2-(2-(pyridin-2-yl)vinyl)-4H-pyran-4-one (2f): The compound $\mathbf{2 f}(83 \mathrm{mg})$ was obtained according to the above general procedure from the compound $3(200 \mathrm{mg}, 0.47$ mmol). Yield: $82 \% ;{ }^{1} \mathrm{H}$ NMR (DMSO- $\left.d_{6}\right) \delta 8.78(\mathrm{dd}, 1 \mathrm{H}, J=$ $6.2,0.8 \mathrm{~Hz}$, pyridin- $H 6), 8.23(\mathrm{~m}, 1 \mathrm{H}$, pyridin- $H 4), 8.16(\mathrm{~s}, 1 \mathrm{H}$, 
pyrone- $H 3), 8.03(\mathrm{~d}, 1 \mathrm{H}, J=8.0 \mathrm{~Hz}$, pyridin- $H 3), 7.68(\mathrm{~m}$, $1 \mathrm{H}$, pyridin- $H 5), 7.63(\mathrm{~d}, 1 \mathrm{H}, J=15.6 \mathrm{~Hz},-\mathrm{CH}=\mathrm{CH}-), 7.46(\mathrm{~d}$, $1 \mathrm{H}, J=15.6 \mathrm{~Hz},-\mathrm{CH}=\mathrm{CH}-), 6.69(\mathrm{~s}, 1 \mathrm{H}$, pyrone- $H 6) ;{ }^{13} \mathrm{C} \mathrm{NMR}$ $\left(\mathrm{DMSO}-d_{6}\right) \delta 174.6,160.1,151.2,147.1,142.1,140.3,130.2$, 127.6, 125.8, 125.1, 115.1.

(E)-2-(2-(Furan-2-yl)vinyl)-5-hydroxy-4H-pyran-4-one (2g): The compound $\mathbf{2 g}$ (20 $\mathrm{mg}$ ) was obtained according to the above general procedure from the compound $\mathbf{3}(100 \mathrm{mg}, 0.30$ mmol). Yield: $32 \%$; ${ }^{1} \mathrm{H}$ NMR (DMSO- $\left.d_{6}\right) \delta 7.92$ (s, $1 \mathrm{H}$, pyroneH6), 7.17 (d, 1H, $J=15.9 \mathrm{~Hz},-\mathrm{CH}=\mathrm{CH}-), 7.09$ (d, $1 \mathrm{H}, J=8.4$ $\mathrm{Hz}$, furan- $H 5), 6.74(\mathrm{~m}, 2 \mathrm{H}$, furan- $H, 34), 6.63(\mathrm{~d}, 1 \mathrm{H}, J=15.9$ $\mathrm{Hz},-\mathrm{CH}=\mathrm{CH}-), 6.55(\mathrm{~s}, 1 \mathrm{H}$, pyrone- $H 3) ;{ }^{13} \mathrm{C}$ NMR (DMSO- $d_{6}$ ) $\delta 174.3,161.2,158.2,156.4,139.2,130.0,127.9,122.5,116.3$, 115.7, 112.0, 109.7.

(E)-5-Hydroxy-2-(2-(thiophen-2-yl)vinyl)-4H-pyran-4-one (2h): The compound $\mathbf{2 h}(70 \mathrm{mg})$ was obtained according to the above general procedure from the compound 3 (200 mg, 0.58 mmol). Yield: $54 \%$; ${ }^{1} \mathrm{H}$ NMR (DMSO- $\left.d_{6}\right) \delta 8.07$ (s, $1 \mathrm{H}$, pyroneH6), $7.70(\mathrm{~d}, 1 \mathrm{H}, J=5.1 \mathrm{~Hz}, \mathrm{Ar}-H 5), 7.60(\mathrm{~d}, 1 \mathrm{H}, J=16.1 \mathrm{~Hz}$, $-\mathrm{CH}=\mathrm{CH}-), 7.46$ (d, $1 \mathrm{H}, J=3.4 \mathrm{~Hz}, \mathrm{Ar}-H 3), 7.16$ (m, $1 \mathrm{H}, \mathrm{Ar}-$ H4), $6.80(\mathrm{~d}, 1 \mathrm{H}, J=16.1 \mathrm{~Hz},-\mathrm{CH}=\mathrm{CH}-), 6.56$ (s, $1 \mathrm{H}$, pyrone$H 3) ;{ }^{13} \mathrm{C}$ NMR (DMSO- $\left.d_{6}\right) \delta 174.3,163.5,161.0,146.3,140.5$, $139.9,139.4,130.8,128.9,128.4,127.4,126.3,119.0,113.3$, 112.1 .

Cell culture and sample treatment. The RAW 264.7 macrophage cell line was obtained from the Korea Cell Line Bank (Seoul). Cells were grown at $37{ }^{\circ} \mathrm{C}$ in DMEM medium supplemented with $10 \%$ FBS, penicillin (100 units $/ \mathrm{mL})$, and streptomycin sulfate $(100 \mu \mathrm{g} / \mathrm{mL})$ in a humidified $5 \% \mathrm{CO}_{2}$ atmosphere. Cells were incubated with various concentrations of tested samples or with positive controls (L-NIL or NS-398) and then stimulated with LPS $1 \mu \mathrm{g} / \mathrm{mL}$ for the indicated time.

Nitrite determination. RAW 264.7 cells were plated at $4 \times 10^{5}$ cells/well in 24 well-plates and then incubated with or without LPS $(1 \mu \mathrm{g} / \mathrm{mL})$ in the absence or presence of various concentrations $(3.15,6.25,12.5,25,50$ and $100 \mu \mathrm{M})$ of tested samples for $24 \mathrm{~h}$. Nitrite levels in culture media were determined using the Griess reaction and presumed to reflect NO levels (Won et al., 2006). Briefly, $100 \mu \mathrm{L}$ of cell culture medium was mixed with $100 \mu \mathrm{L}$ of Griess reagent [equal volumes of $1 \%(\mathrm{w} / \mathrm{v})$ sulfanilamide in $5 \%(\mathrm{v} / \mathrm{v})$ phosphoric acid and $0.1 \%(\mathrm{w} / \mathrm{v})$ naphtylethylenediamine- $\mathrm{HCl}]$, and incubated at room temperature for $10 \mathrm{~min}$. Absorbance was then measured at $540 \mathrm{~nm}$ using a microplate reader (Perkin Elmer Cetus, Foster City, CA, USA). Fresh culture media were used as blanks in all experiments. The amount of nitrite in the samples was measured with the sodium nitrite serial dilution standard curve and nitrite production was measured.

PGE 2 assay. RAW 264.7 cells were pretreated with tested samples for $1 \mathrm{~h}$ and then stimulated with LPS $(1 \mu \mathrm{g} / \mathrm{mL})$ for $24 \mathrm{~h}$. Levels of $\mathrm{PGE}_{2}$ in the culture media was quantified using EIA kits (R\&D Systems, Minneapolis, MN. USA) according to the manufacturer's instructions.

MTT assay for cell viability. RAW 264.7 cells were plated at a density of $10^{5}$ cells/well in 96 -well plates. To determine the appropriate concentration not toxic to cells, cytotoxicity studies were performed $24 \mathrm{~h}$ after treating cells with various concent rations of tested compounds. Viabilities were determined using colorimetric MTT assays, as described previously. ${ }^{27}$

Acknowledgments. This work was supported by the Basic Science Research Program through the National Research Foundation of Korea (NRF) funded by MEST (2010-0016080).

\section{References}

1. Oberyszyn, T. M. Front. Biosci. 2007, 12, 2993-2999.

2. Vane, J. R.; Mitchell, J. A.; Appleton, I.; Tomlinson, A.; BishopBailey, D.; Croxtall, J.; Willoughby, D. A. Proc. Natl. Acad. Sci. USA. 1994, 91, 2046-2050.

3. Yun, H. Y.; Dawson, V. L.; Dawson, T. M. Crit. Rev. Neurobiol. 1996, 10, 291-316.

4. Hinz, B.; Brune, K. J. Pharmacol. Exp. Ther. 2002, 300, 367-375.

5. Burns, J.; Yokota, T.; Ashihara, H.; Lean, M. E. J.; Crozier, A. J. Agric. Food. Chem. 2002, 50, 3337-3340.

6. Rimando, A. M.; Kalt, W.; Magee, J. B.; Dewey, J.; Ballington, J. R. J. Agric. Food. Chem. 2004, 52, 4713-4719.

7. Sanders, T. H.; McMichael, R. W., Jr.; Hendrix, K. W. J. Agric. Food. Chem. 2000, 48, 1243-1246.

8. Jang, M.; Cai, L.; Udeani, G. O.; Slowing, K. V.; Thomas, C. F.; Beecher, C. W.; Fong, H. H.; Farnsworth, N. R.; Kinghorn, A. D.; Mehta, R. G.; Moon, R. C.; Pezzuto, J. M. Science 1997, 275, 218-220.

9. Subbaramaiah, K.; Chung, W. J.; Michaluart, P.; Telang, N.; Tanabe, T.; Inoue, H.; Jang, M.; Pezzuto, J. M.; Dannenberg, A. J. J. Biol. Chem. 1998, 273, 21875-21882.

10. Tsai, S. H.; Lin-Shiau, S. Y.; Lin, J. K. Br. J. Pharmacol. 1999, 126 , 673-680.

11. Bradamante, S.; Barenghi, L.; Villa, A. Cardiovasc. Drug Rev. 2004, 22, 169-188.

12. Trela, B. C.; Waterhouse, A. L. J. Agric. Food Chem. 1996, 44, 1253-1257.

13. Bi, X. L.; Yang, J. Y.; Dong, Y. X.; Wang, J. M.; Cui, Y. H.; Ikeshima, T.; Zhao, Y. Q.; Wu, C. F. Int Immunopharmacol. 2005, 5, $185-93$.

14. Cho, D.-I.; Koo, N.-Y.; Chung, W. J.; Kim, T.-S.; Ryu, S. Y.; Im, S. Y.; Kim, K.-M. Life Sciences 2002, 71, 2071-2082.

15. Heynekamp, J. J.; Weber, W. M.; Hunsaker, L. A.; Gonzales, A. M.; Orlando, R. A.; Deck, L. M.; Vander Jagt, D. L. J. Med. Chem. 2006, 49, 7182-7189.

16. Kang, S. S.; Cuendet, M.; Endringer, D. C.; Croy, V. L.; Pezzuto, J. M.; Lipton, M. A. Bioorg. Med. Chem. 2009, 17, 1044-1054.

17. Meng, X. L.; Yang, J. Y.; Chen, G. L.; Zhang, L. J.; Wang, L. H.; Li, J.; Wang, J. M.; Wu, C. F. Int Immunopharmacol. 2008, 8, 10741082.

18. Meng, X.-L.; Yang, J.-Y.; Chen, G.-L.; Wang, L.-H.; Zhang, L.-J.; Wang, S.; Li, J.; Wu, C.-F. Chemico-Biol. Interact. 2008, 174, 51-59.

19. Park, J. H.; Min, H.-Y.; Kim, S. S.; Lee, J. Y.; Lee, S. K.; Lee, Y. S. Arch. Pharm. 2004, 337, 20-24.

20. Kang, S. S.; Kim, H. J.; Jin, C.; Lee, Y. S. Bioorg. Med. Chem. Lett. 2009, 19, 188-191.

21. Lion, C. J.; Matthews, C. S.; Stevens, M. F. G.; Westwell, A. D. J. Med. Chem. 2005, 48, 1292-1295.

22. Lesk, A.; Nudelman, A. Synth. Commun. 1999, 29, 1405-1408.

23. Rumbo, A.; Mourino, A.; Castedo, L.; Mascarenas, J. L. J. Org. Chem. 1996, 61, 6114-6120.

24. Wright, J. A.; Yu, J.; Spencer. J. B. Tetrahedron Lett. 2001, 42, 4033-4036.

25. Horita, K.; Yoshioka, T.; Tanaka, T.; Oikawa, Y.; Yonemitsu, O. Tetrahedron 1986, 42, 3021-3028.

26. Paliakov, E.; Strekowski, L. Tetrahedron Lett. 2004, 45, 40934095.

27. Kim, J. Y.; Park, S. J.; Yun, K. J.; Cho, Y. W.; Park, H. J.; Lee, K. T. Eur. J. Pharmacol. 2008, 584, 175-184. 